\title{
A Switched Control Strategy for Swing-Up and State Regulation for the Rotary Inverted Pendulum
}

\author{
Miguel A. SOLIS ${ }^{1 *}$, Manuel OLIVARES ${ }^{2}$, Héctor ALLENDE ${ }^{3}$ \\ ${ }^{1}$ Department of Computer \& Systems Engineering, Universidad Católica del Norte Avenida Angamos 0610, \\ Antofagasta, Chile \\ miguel.solis@ucn.cl (*Corresponding author) \\ ${ }^{2}$ Department of Electronics Engineering, Universidad Técnica Federico Santa María Avenida España 1680, \\ Valparaíso, Chile \\ manuel.olivares@usm.cl \\ ${ }^{3}$ Department of Informatics, Universidad Técnica Federico Santa María Avenida España 1680, \\ Valparaíso, Chile \\ hector.allende@usm.cl
}

\begin{abstract}
The rotary inverted pendulum (RIP) is an underactuated mechanical system that can be balanced in the upward position, by applying the linear control theory based on a nonlinear dynamical model linearized around that position. Its structure makes it possible to implement controllers designed for energy minimization in the non-linearizable region. Due to the limited validity of the resulting linear model, a nonlinear control is needed to automatically move the pendulum close to the linearized region, starting from its stable downward position. A RIP platform has been built to experimentally test a switched control strategy design, which combines an energy-based nonlinear controller for swinging up the pendulum and a linear quadratic regulator for the balancing task. Experimental results validate the performance of this approach on the physical prototype.
\end{abstract}

Keywords: Linear quadratic regulator, Energy-based control, Lyapunov stability, Rotary inverted pendulum, Underactuated systems.

\section{Introduction}

Underactuated systems (Spong, 1998), (Fantoni \& Lozano, 2002) pose challenging control and implementation problems in broad application areas such as aerospace systems, robotic platforms and mechatronic systems, among others, due to their limited control inputs for reaching multiple objectives, their nonlinear dynamics and their dynamic and physical constraints. There are several approaches for solving them, which range from model-based nonlinear control to model-free intelligent control.

The development of underactuated mechatronic systems as benchmark problems for education and research, has enabled the testing of different control approaches. For example, the cart-pole inverted pendulum benchmark requires not only balancing the pendulum in the upward unstable position, but also taking into account the restrictions on the cart displacement, as in (Yu, Liu \& Tang, 2008), thus increasing design complexity. Radial basis function neuronal networks are included to maintain the balance of an inverted pendulum while tracking a desired position of the mobile cart in (Noh, Lee \& Jung, 2010), and a decentralized neural network control is applied in (Jung \& Cho, 2004) to control both the pendulum angle and the position of the cart mounted in an $\mathrm{x}-\mathrm{y}$ table.
Switched systems are based on a set of subsystems and a switching signal selecting a subsystem to be active during given circumstances such as an interval of time, or a given region in the appropriate space of the respective application (Liberzon, 2003). This kind of systems has been extensively used in applications such as aircraft and air traffic control or mechanical systems (Zhang \& Tarn, 2003). The latter is also a relevant area for underactuated systems such as the ones described in this paper, whose control strategy combines a linear stabilization controller when the linear model of the plant is active, and a nonlinear controller with energy minimization criteria when it is not active.

The rotary inverted pendulum (RIP) is an underactuated system with two degrees of freedom and one actuator. It is also known as the Furuta pendulum (Åström \& Furuta, 2000), and it has also been widely considered for testing. Different nonlinear control design approaches for the Furuta pendulum can be found in literature, such as in (Zehar, Benmahammed \& Behih, 2018) where the design and implementation of an adaptive sliding mode controller is discussed, along with other sliding mode variations in order to compare their performance within that class of controllers. 
Fuzzy logic controllers and other intelligent control techniques such as swing-up control by using trajectory planning and stabilization using artificial neural networks when the model is described through linear matrix inequalities, are discussed in (Yang \& Zheng, 2018). Work in (Hassanzadeh \& Mobayen, 2011) presents the use of evolutionary algorithms for PID control parameters tuning, where genetic algorithms, particle swarm optimization and ant colony optimization are applied. These methods may improve the performance of the obtained controller, but require additional analysis to guarantee stability and convergence speed in the intermediate process.

Although nonlinear control does not deal with model approximation problems such as approximation errors or underfitting parameters, linear control is a powerful approach which can enable a simpler stability analysis and control design due to the extensively studied linear theory. For instance, a linear model predictive control for the pendulum balance is combined with swing-up strategies in (Seman et al., 2013), and the work in (Ramírez-Neria et al., 2014) present a linear controller whose input is based on a linear observer, with a control strategy obtained under an active disturbance rejection control (ADRC) scheme.

The flywheel inverted pendulum (FIP), another underactuated mechanical system, has been analysed in (Block, Åström \& Spong, 2007) where the design complexity is reduced using a linearized model to design a linear control for balancing the pendulum, combined with energy-based strategies for swing-up. (Olivares \& Albertos, 2014a) present an energy-based nonlinear controller design for the swing-up task of the FIP, in combination with a locally stabilizing PID control strategy designed to balance the pendulum in the upward unstable position with user-defined flywheel velocity reference, while measuring disturbance compensation, as detailed in (Olivares \& Albertos, 2014b).

In this paper, the results for the flywheel inverted pendulum are extended to the RIP, providing a more in-depth analysis of the switched control strategy, which involves commuting from the local stabilizing controller to the nonlinear swing-up controller. Thereby, a state feedback Linear Quadratic Regulator (LQR) strategy is designed instead of a PID control strategy for pendulum balance. Firstly, the RIP nonlinear model is obtained based on a Lagrangian approach, and it is linearized around the unstable position, where $\sin (\theta) \sim \theta$. The switch strategy presented consists in an energy-based swing-up task, unlike other approaches found in literature such as the one which focuses on a bang-bang swing-up controller as presented by (Park, Kim \& Lee, 2011). The latter could be considered as the closest approach to this proposal since LQR is also applied for stabilization, but with another type of swing-up controller, as well as a faster sampling period which requires more resources for the data acquisition task. The main contribution of this work is the control of both degrees of RIP freedom, allowing the user to define the rotating arm position and to balance the inverted pendulum simultaneously. It also includes experimental results on a nonlinear underactuated physical prototype, where due to the chosen state space representation, the current applied to the motor in addition to pendulum and rotating arm position and velocities, could also be diminished through appropriate control parameters.

The paper is organized as follows. Section 2 presents the RIP nonlinear model along with its linear approximation around the unstable equilibrium point, and its validation using measured data related to the physical prototype. Section 3 sets forth the energy-based nonlinear controller for the swing-up task, which was created based on the Lyapunov global stability theory. Then, Section 4 formulates the LQR problem which leads to a state feedback control law in order to balance the pendulum in the upward unstable position, while Section 5 presents experimentally obtained results on the switched strategy. Finally, Section 6 presents some final conclusions and future work is outlined.

\section{The Rotary Inverted Pendulum}

Consider a second order and controllable dynamical system given by:

$\ddot{q}=f(q, \dot{q}, u, t)$,

https://www.sic.ici.ro 
where $u$ corresponds to the (scalar) control input, $t$ denotes the possible influence of time on the acceleration vector $\ddot{q}$, and $q$ and $\dot{q}$ are the positions and velocity vectors respectively, which are described by:

$$
\begin{aligned}
& q=\left[\begin{array}{ll}
\theta_{0} & \theta_{1}
\end{array}\right]^{T}, \\
& \dot{q}=\left[\begin{array}{ll}
\dot{\theta}_{0} & \dot{\theta}_{1}
\end{array}\right]^{T},
\end{aligned}
$$

with $\theta_{0}$ being the arm rotation angle, and $\theta_{1}$ representing the pendulum inclination as depicted in Figure 1.

For the case presented in this paper, where commanded torque is linear in the dynamical model, this expression can be rewritten as:

$\ddot{q}=f_{1}(q, \dot{q}, t)+f_{2}(q, \dot{q}, t) \mathrm{u}$.

Then, in formal terms a dynamical system described by (1) is called underactuated in the configuration $(q, \dot{q}, t)$ if it is not possible to generate an instantaneous acceleration in any arbitrary direction, i.e.:

$$
\operatorname{rank}\left(f_{2}(q, \dot{q}, t)\right)<\operatorname{dim}(u)
$$

The rotary inverted pendulum (RIP) (Furuta, Yamakita \& Kobayashi, 1992), also known as the Furuta pendulum is an example of an underactuated system. As illustrated in Figure 1, it consists in a controlled arm on the horizontal plane rotating around a central axis, and a pendulum linked to one of the ends of this arm, rotating over the vertical plane. The nonlinear model of this plant allows the study of nonlinear dynamics, which also makes it possible to construct it physically as a mechatronic platform with enhanced reproducibility features for instrumentation and the implementation of control systems.

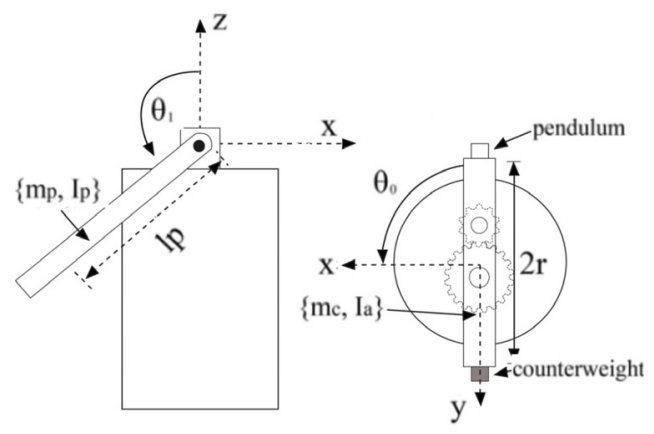

Figure 1. Side and top view of Furuta pendulum

According to Figure 1, there is a direct current (DC) motor responsible for controlling the arm position measured by $\theta_{0}$ through the armature voltage, and its main parameters are the armature resistance and inductance, $R_{a}$ and $L_{a}$ respectively.

\subsection{Model Formulation}

Define $\left\{l_{p}, m_{p}, I_{p}, \theta_{1}\right\}$, where $l_{p}$ stands for the pendulum length from the rotating axis to its center of mass, $m_{p}$ denotes the mass of the pendulum with its moment of inertia $I_{p}$ and its rotation angle $\theta_{1}$. Then, $r$ corresponds to the arm radius which has a moment of inertia $I_{a}$, and a counterweight of mass $m_{c}$ which takes the center of the mass of the rotating arm at height $h$ is introduced. Consider the rotation angle of the arm to be given by $\theta_{0}$. A detailed description of the parameters involved in the model along with their values for the physical prototype is illustrated in Table 1.

The Lagrangian formulation is then given by (5), where $E_{k}$ and $E_{p}$ denote the kinetic and potential energy respectively, while $q$ is the generalized coordinates' vector, $q=\left[\begin{array}{ll}\theta_{0} & \theta_{1}\end{array}\right]^{T}$.

$L(q, \dot{q})=E_{k}(q, \dot{q})-\mathrm{E}_{p}(q, \dot{q})$.

Kinetic energy for the pendulum is given by the sum of traslational and rotational components, while kinetic energy for the arm is given by rotation and tangential kinetic energy:

$$
\begin{aligned}
E_{k}= & \frac{1}{2} \hat{J}_{0} \dot{\theta}_{0}^{2}+\frac{1}{2} \hat{J}_{1} \dot{\theta}_{1}^{2}+\frac{1}{2} m_{p} l_{p}^{2} \dot{\theta}_{0}^{2} \sin ^{2}\left(\theta_{1}\right) \\
& -m_{p} r l_{p} \dot{\theta}_{0} \dot{\theta}_{1} \cos \left(\theta_{1}\right)
\end{aligned}
$$

where $\hat{J}_{0}$ and $\hat{J}_{1}$ are given by

$\hat{J}_{0}=I_{a}+r^{2} \cdot\left(m_{p}+m_{c}\right)$

$\hat{J}_{1}=I_{p}+m_{p} l_{p}^{2}$

while potential energy is given in terms of the pendulum and counterweight masses:

$E_{p}=m_{p} g l_{p} \cdot \cos \left(\theta_{1}\right)$

Then, according to the Euler-Lagrange equation:

$\frac{d}{d t}\left(\frac{\partial L}{\partial \dot{q}_{i}}\right)-\frac{\partial L}{\partial q_{i}}=\tau_{i} \quad, \quad i=\theta_{0}, \theta_{1}$

with $\tau_{i}$ being the moments applied to each coordinate, leading to

$$
M\left(\theta_{1}\right) \ddot{q}+V\left(\theta_{1}\right) \dot{q}+G\left(\theta_{1}\right)=T,
$$

where $M$ corresponds to inertia, $V$ to centripetal and Coriolis torque and $G$ to gravitational torque matrices respectively, and $T$ is the torque vector. It can be noticed that although $\theta_{0}$ does not appear explicitly in this last expression, its velocity and 
Table 1. Physical parameters of the RIP prototype

\begin{tabular}{|c|c|c|}
\hline Symbol & Description & Value \\
\hline$m_{p}$ & Pendulum mass $[\mathrm{kgs}]$ & 0.1 \\
\hline$m_{c}$ & Counterweight mass $[\mathrm{kgs}]$ & 0.01 \\
\hline$I_{p}$ & Pendulum inertial moment $\left[\mathrm{kgs} \cdot \mathrm{mts}^{2}\right]$ & $5.1 \cdot 10^{-4}$ \\
\hline$I_{a}$ & Arm inertial moment $\left[\mathrm{kgs} \cdot \mathrm{mts}{ }^{2}\right]$ & $3.1 \cdot 10^{-3}$ \\
\hline$r$ & Arm radius $[\mathrm{mts}]$ & 0.13 \\
\hline$l_{p}$ & Pendulum mass center $[\mathrm{mts}]$ & 0.125 \\
\hline$C_{0}$ & Arm friction coefficient $[\mathrm{N} \cdot \mathrm{mts} \cdot \mathrm{sec}]$ & $10^{-4}$ \\
\hline$C_{1}$ & Pendulum friction coefficient $[\mathrm{N} \cdot \mathrm{mts} \cdot \mathrm{sec}]$ & $10^{-4}$ \\
\hline$R_{a}$ & Armature resistor $[\Omega]$ & 10 \\
\hline$L_{a}$ & Motor inductance $[\mathrm{mH}]$ & $1.9 \cdot 10^{-6}$ \\
\hline$I_{m}$ & Motor inertia $\left[\mathrm{kgs} \cdot \mathrm{mts}{ }^{2}\right]$ & 0.0214 \\
\hline$M_{f}$ & Motor mutual inductance $[\mathrm{N} \cdot \mathrm{mts} / \mathrm{A}]$ & 59927 \\
\hline$K_{g}$ & Motor gear reduction coefficient & 16 \\
\hline$K_{e g}$ & External gear reduction coefficient & 9.806 \\
\hline$g$ & Gravitational acceleration $\left[\mathrm{mts} / \mathrm{sec}^{2}\right]$ & \\
\hline & & \\
\hline
\end{tabular}

acceleration are included for $\dot{q}$ and $\ddot{q}$ respectively, as well as in the following matrices:

$M\left(\theta_{1}\right)=\left[\begin{array}{cc}\hat{J}_{0}+m_{p} l_{p}^{2} \sin ^{2}\left(\theta_{1}\right) & -m_{p} r l_{p} \cos \left(\theta_{1}\right) \\ -m_{p} r l_{p} \cos \left(\theta_{1}\right) & \hat{J}_{1}\end{array}\right]$,
$V\left(\theta_{1}\right)=\left[\begin{array}{cc}m_{p} l_{p}^{2} \sin \left(2 \theta_{1}\right)+C_{0} & m_{p} r l_{p} \dot{\theta}_{1} \sin \left(\theta_{1}\right) \\ -\frac{1}{2} m_{p} l_{p}^{2} \dot{\theta}_{0} \sin \left(2 \theta_{1}\right) & C_{1}\end{array}\right]$,

$G\left(\theta_{1}\right)=\left[\begin{array}{c}0 \\ -m_{p} g l_{p} \sin \left(\theta_{1}\right)\end{array}\right]$,

$T=\left[\begin{array}{ll}\tau_{l} & 0\end{array}\right]^{T}$,

with $C_{0}$ and $C_{1}$ as the friction coefficients of the arm and pendulum respectively, with the respective values given in Table 1. From (11), it can be noticed that the motor actuates just on the arm and matrix model of the movement transfer to the pendulum.

The input torque, $\tau_{l}$ is related to the motor dynamics which is related to electrical torque, $\tau_{e}$, and electrical angular velocity, $\omega_{e}$, given by
$v(t)=R_{a} i(t)+L_{a} \frac{d}{d t} i(t)+M_{f} \omega_{e}$,

$\tau_{e}=M_{f} K_{g} i(t)$

$\tau_{e}-\tau_{l}=I_{m} \frac{d}{d t} \omega_{e}$

with parameters as described in Table 1, and $v(t)$ and $i(t)$ are the voltage and the current applied to the motor at time $t$, respectively.

Finally, the applied torque and angular velocity are related to their corresponding electrical variables as follows:

$\omega_{0}=K_{g}^{-1} \omega_{e}$

$\tau_{0}=K_{g} \tau_{e}$

From (13a) and noting that this prototype has an external gear reduction system which relates the arm angular velocity to its corresponding variable obtained from the motor described by

$\dot{\theta}_{0}=K_{e g}^{-1} \omega_{0}$, 
expression (12a) can be rewritten as

$$
\frac{d}{d t} i(t)=\frac{1}{L_{a}} v(t)-\frac{R_{a}}{L_{a}} i(t)-\frac{M_{f}}{L_{a} K_{g}} \dot{\theta}_{0}
$$

Then, the RIP model including motor dynamics is given by:

$\ddot{q}=\bar{M}\left(\theta_{1}\right)^{-1}\left(\bar{T}-\bar{V}\left(\theta_{1}\right) \dot{q}-G\left(\theta_{1}\right)\right)$

where new matrices are described by:

$\bar{M}\left(\theta_{1}\right)=\left[\begin{array}{cc}\hat{J}_{0}+m_{p} l_{p}^{2} \sin ^{2}\left(\theta_{1}\right)+K_{g}^{2} I_{m} & -m_{p} r l_{p} \cos \left(\theta_{1}\right) \\ -m_{p} r l_{p} \cos \left(\theta_{1}\right) & \hat{J}_{1}\end{array}\right]$,

$\bar{V}\left(\theta_{1}\right)=\left[\begin{array}{cc}m_{p} l_{p} \dot{\theta}_{1} \sin \left(2 \theta_{1}\right)+C_{0} & m_{p} r l_{p} \dot{\theta}_{1} \sin \left(\theta_{1}\right) \\ -\frac{1}{2} m_{p} l_{p} \dot{\theta}_{0} \sin \left(2 \theta_{1}\right) & C_{1}\end{array}\right]$,

$\bar{T}=\left[\begin{array}{ll}M_{f} K_{g} i & 0\end{array}\right]^{T}$,

noting that according to the definition in (3), such functions are related to the plant model as follows:

$$
\begin{aligned}
& f_{1}(q, \dot{q}, t)=-\overline{\mathrm{M}}\left(\theta_{1}\right)^{-1}\left(\bar{V}\left(\theta_{1}\right) \dot{q}+G\left(\theta_{1}\right)\right), \\
& f_{2}(q, q, t) u=\bar{M}\left(\theta_{1}\right)^{-1} \bar{T}
\end{aligned}
$$

where $q$ is implicitly defined through the presence of $\theta_{0}$ and $\theta_{1}$, and actuation $u$ appears in the form of the applied current to the motor, $i$, in $\bar{T}$.

Then, the state vector $x=\left[\begin{array}{lllll}\theta_{0} & \dot{\theta}_{0} & \theta_{1} & \dot{\theta}_{1} & i\end{array}\right]^{T}$ is defined. Then, in order to obtain a linearized model around the equilibrium point $x=\left[\begin{array}{lllll}0 & 0 & 0 & 0 & 0\end{array}\right]$, the derivative of current $i$ is solved based on equation (12a) :

$\frac{d}{d t} i(t)=\frac{1}{L_{a}} v(t)-\frac{R_{a}}{L_{a}} i(t)-\frac{M_{f}}{L_{a}} \omega_{e}$

Then, the following linear model is obtained

$\dot{x}(t)=A x(t)+B u(t)$,

where $x$ is the previously defined state vector, $u$ corresponds to the control signal (applied voltage) and matrices $A$ and $B$ are given by (21) and (22) respectively,

$$
A=\alpha\left[\begin{array}{ccccc}
0 & \frac{1}{\alpha} & 0 & 0 & 0 \\
0 & -\hat{J}_{1} C_{0} & m_{p}^{2} l_{p}^{2} g r & m_{p} r l_{p} C_{1} & M_{f} K_{g} \hat{J}_{0} \hat{J}_{1} \\
0 & 0 & 0 & \frac{1}{\alpha} & 0 \\
0 & m_{p} r l_{p} C_{0} & \hat{J}_{0} m_{p} g l_{p} & -\hat{J}_{0} C_{1} & K_{g} M_{f} m_{p} r l_{p} \\
0 & -\frac{M_{f}}{\alpha L_{a} K_{g}} & 0 & 0 & -\frac{R_{a}}{\alpha L_{a}}
\end{array}\right]
$$

$B=\left[\begin{array}{lllll}0 & 0 & 0 & 0 & \frac{1}{L_{a}}\end{array}\right]^{T}$,

and $\alpha$ is given by the following expression:

$$
\alpha=\frac{1}{\hat{J}_{0} \hat{J}_{1}-\left(m_{p} r l_{p}\right)^{2}}
$$

with eigenvalues in

$$
\left\{\begin{array}{lllll}
-772.36 & -28.06 & -7.87 & 0 & 7.48
\end{array}\right\} .
$$

The internal instability generated by the pole in the point of origin and by another one in the righthalf plane can be noticed, also observing that in case the arm position were not a matter of interest in the control task, matrix $A$ could be reduced to 4 rows and 4 columns because of null values in its first column. This shows that variables are affected only by arm velocity, pendulum position and velocity, and the current injected into the motor.

\subsection{Model Validation}

Considering a region of 15 degrees around the downward stable position, $\theta_{1}=\pi$, the dynamic equation for the pendulum can be approximated as linear and can be written as:

$\hat{J}_{1} \ddot{\theta}_{1}+C_{1} \dot{\theta}_{1}+m_{p} l_{p} g \theta_{1}=0$

or in its general form:

$\ddot{\theta}_{1}+2 \zeta \omega_{n} \dot{\theta}_{1}+\omega_{n}^{2} \theta_{1}=0$

with $\omega_{n}$ as the natural oscillation frequency and $\zeta$ as the damping coefficient. From (24) and (25) we have:

$\omega_{n}=7.69$

$\zeta=3.13 \cdot 10^{-3}$.

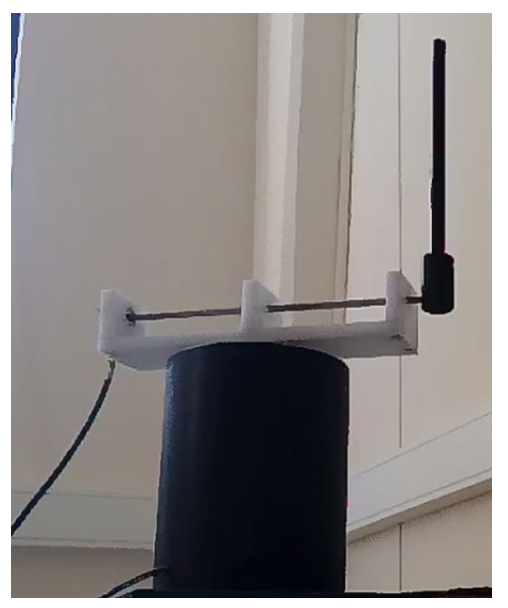

Figure 2. Physical prototype of the Furuta Pendulum 
Experimentally based on the physical prototype shown in Figure 2, these parameters can be estimated from a free response of the system as:

$$
\begin{aligned}
& \hat{\zeta}=\frac{1}{\sqrt{1+\left(\frac{\pi}{\ln ^{2}(K / N)}\right)}}, \\
& \hat{\omega}_{n}=\frac{2 \pi}{T_{s} \sqrt{1-\hat{\zeta}^{2}}},
\end{aligned}
$$

where $K$ corresponds to the ratio from the first two consecutive peaks, $N$ is the stationary value and $T_{s}$ stands for the semi-period of the maximum oscillation. Then, from Figure 3 the following is obtained:

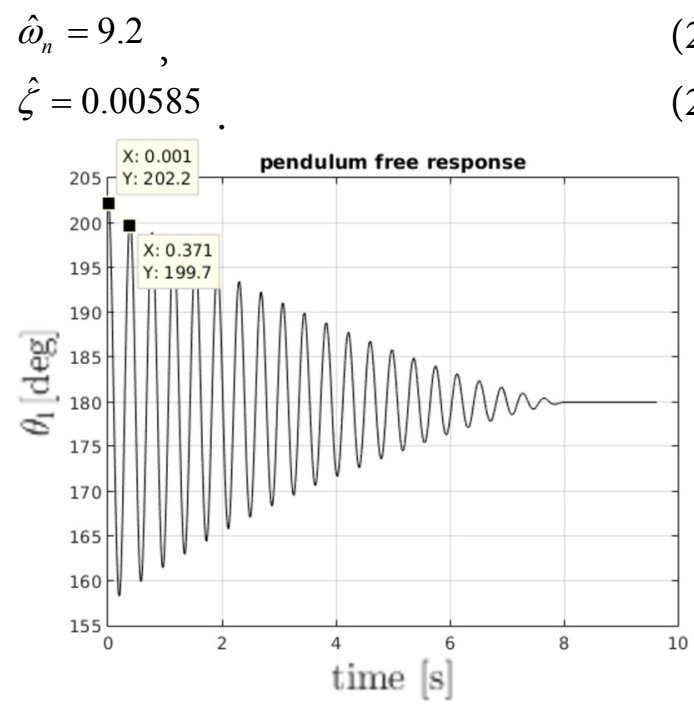

Figure 3. Pendulum damped oscillation

The reader should note that in the following sections, in particular for state-feedback implementation, it is required to use velocity sensors in order to make appropriate measurements. Given that minimization of physical wires around the plant is desirable in case of wired-sensors for avoiding roll-ups (or the minimization of time delays when considering wireless measurements), a linear observer $O$ is used for estimating velocities on arm and pendulum, with a bandwidth which enables it to detect changes among samples when acquiring data, and whose (continuous) transfer function is given by

$O(s)=\frac{50 s}{s+50}$

\section{The Swing-up Controller}

The model from (16) can be simplified if we do not consider the centripetal and Coriolis torque, that is by inserting null values in order to replace the current entries of matrices $V\left(\theta_{1}\right)$ and $G\left(\theta_{1}\right)$, thus obtaining:

$\hat{J}_{1} \ddot{\theta}_{1}-m_{p} g l_{p} \sin \left(\theta_{1}\right)=m_{p} r \ddot{\theta}_{0} \cos \left(\theta_{1}\right)$

Then, taking into account that the angular acceleration $\theta_{0}$ is directly controlled from the actuator, the total unforced energy for the pendulum is given by:

$$
E_{u}=\frac{1}{2} \hat{J}_{1} \dot{\theta}_{1}^{2}+m_{p} g l_{p} \cos \left(\theta_{1}\right)+m_{c} g h .
$$

Given that the objective of the switched control strategy is to stabilize the pendulum in the upward position, that is when $\theta_{1}=\dot{\theta}_{1}=0$, the desired energy for the target position is given by:

$E_{d}=m_{p} g l_{p}+m_{c} g h$

which implied that the unforced normalized energy in relation to $E_{d}$ is as follows:

$E_{u n}=E_{u}-E_{d}$,

$=\frac{1}{2} \hat{J}_{1} \dot{\theta}_{1}^{2}+m_{p} g l_{p}\left(\cos \left(\theta_{1}\right)-1\right)$

leading to $E_{u n}=0$ for the equilibrium position and $E_{u n}=-2 m_{p} g l_{p}$ at the rest (downward) position.

Figure 4 shows the evolution of unforced normalized energy, $E_{u n}$, over time when the pendulum moves from the upward to the rest position with no control signal being applied on the system.
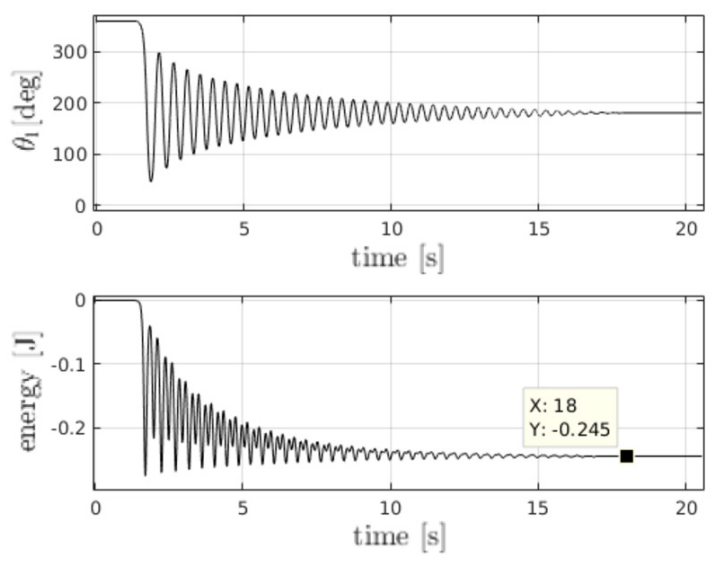

Figure 4. Unforced normalized energy evolution 
In order to obtain a nonlinear controller that minimizes (33), the following positive definite Lyapunov function should be considered:

$U=\frac{1}{2} E_{u n}^{2}$

Then, a controller minimizing (33), that is taking $E_{u n} \rightarrow 0$ asymptotically as $t \rightarrow \infty$ from any given initial condition, has to be chosen such that $\dot{U}<0$. This derivative is given by:

$\dot{U}=\left(E_{u}-E_{d}\right) \dot{E}_{u}$,

where by using (31) and (32), and based on the fact that $\dot{E}_{u n}=\dot{E}_{u}$ since $E_{d}$ is constant, the derivative of unforced energy is obtained:

$$
\dot{E}_{u}=m_{p} r \ddot{\theta}_{0} \cos \left(\theta_{1}\right) \dot{\theta}_{1} \text {. }
$$

Therefore, the proposed control law generates a positive swing-up gain, $\lambda_{s u}$ whose value is chosen such that (35) is never positive semi-definite:

$\ddot{\theta}_{0}=-\lambda_{s u}\left(E_{u}-E_{d}\right) \cos \left(\theta_{1}\right) \dot{\theta}_{1}$.

It can be noticed that in order to achieve a given arm acceleration, the expression (37) should be integrated and defined in relation to the arm angular velocity.

As controlling the angular velocity of the motor implies controlling its current, the transfer function from current to voltage is simplified by neglecting armature inductance, so the relation between angular acceleration and the applied voltage becomes:

$v(t)=R_{a} i(t)+\mathrm{M}_{f} \omega_{e}$.

On the other hand, considering (12b) and

$\tau_{l} \approx \hat{J}_{0} \ddot{\theta}_{0}$ into $(12 \mathrm{c})$ :

$K_{g} M_{f} i(t)+\hat{J}_{0} \lambda_{s u}\left(E_{u}-E_{d}\right) \cos \left(\theta_{1}\right) \dot{\theta}_{1}=I_{m} \frac{d}{d t} \omega_{e}$

so by solving (38) for the current and replacing it, and (13a) with (14) into (39), the control law expressed in terms of the applied voltage would be given by

$$
v(t)=M_{f} K_{g} K_{e g} \dot{\theta}_{0}-\bar{\lambda}_{s u}\left(E_{u}-E_{d}\right) \cos \left(\theta_{1}\right) \dot{\theta}_{1},
$$

with

$$
\bar{\lambda}_{s u}=\frac{R_{a}\left(\hat{J}_{0}+I_{m} K_{g} K_{e g}\right)}{K_{g} M_{f}} \lambda_{s u}
$$

Figure 5 shows the pendulum position on the physical platform and the corresponding control signal applied to the motor while performing the swing-up movement.
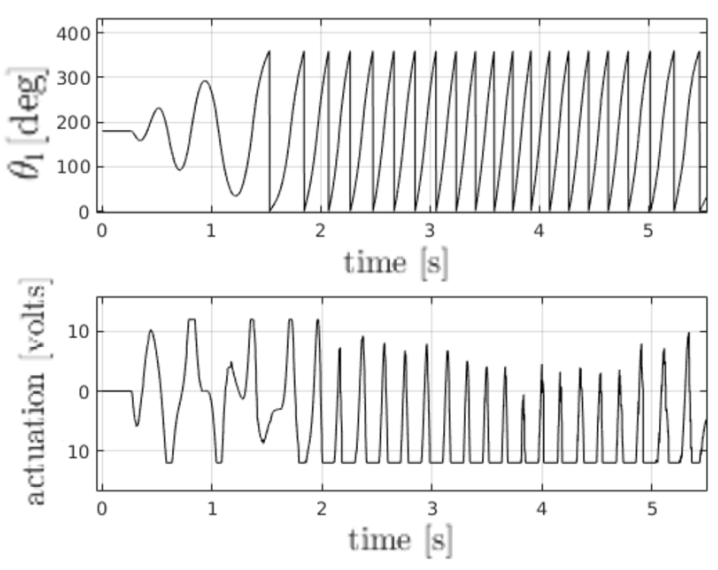

Figure 5. Physical position and actuation in the swing-up

\section{The Linear Quadratic Regulator}

Once the pendulum position was moved from the downward equilibrium state to the upward unstable position, the next step is to keep the pendulum at zero degrees with the minimum possible deviation, so a stabilizing controller has to be obtained. Considering a linear and timeinvariant system described in state space variables as in equation (20), the cost function is given by:

$J=\int_{0}^{\infty}\left(x^{T}(t) Q x(t)+u^{T}(t) R u(t)\right) d t$

where $Q$ and $R$ are weighting matrices that are positive definite and positive semi-definite respectively. Then, the state-feedback control law which minimizes (42) is given by:

$u(t)=r(t)-L x(t)$,

where $r(t)=0$ for this regulator given that the reference value corresponds in this case to the upward unstable position, and $L$ is such that

$L=R^{-1} B^{T} P$,

and $P$ stands for the solution of the algebraic Ricatti equation (ARE):

$A^{T} P+P A-P B R^{-1} B^{T} P+Q=0$.

Matrices have to be designed by means of giving more weight to states that are more relevant than others for a particular experiment. In this case, priority is given to pendulum position, $\theta_{1}$, and also 
to arm and pendulum velocities, so the proposed experiment is given by:

$Q=\left[\begin{array}{ccccc}1 & 0 & 0 & 0 & 0 \\ 0 & 10 & 0 & 0 & 0 \\ 0 & 0 & 100 & 0 & 0 \\ 0 & 0 & 0 & 10 & 0 \\ 0 & 0 & 0 & 0 & 1\end{array}\right]$,

$R=10$,

where the penalizing actuation factor, $R$, is chosen such that DC motor does not perform any sudden behavior changes that would destabilize the pendulum position.

Then, solving (45) with parameters $A$ and $B$ described in (21) and (22), and replacing expression solved in (45) into (44) yields:

$$
L=\left[\begin{array}{lllll}
-0.31 & -5.26 & 70.74 & 8.92 & 0.17
\end{array}\right] \text {. }
$$

This also yields closed-loop poles located at

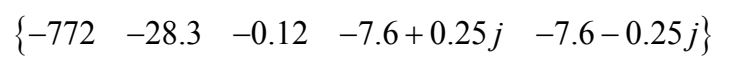

where $j$ denotes the complex component in the complex plane. The closed-loop stability with all its poles in the left-half complex plane can be noticed.

\section{The Switched Control Strategy}

The switched control strategy consists in combining the energy-based swing-up task described in this paper, and the state-feedback controller obtained by solving the linear quadratic regulator problem discussed in Section 3. This involves commuting from the swing-up controller to the LQR control when the pendulum position changes by less than 15 degrees in magnitude, around the upward unstable position, as depicted in Figure 6, where $r$ stands for the reference of the arm position.

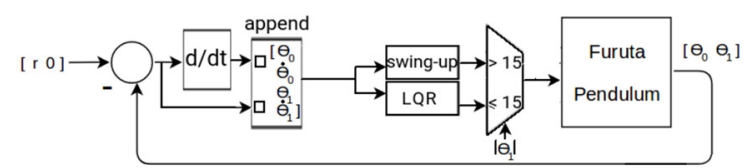

Figure 6. Switched strategy illustrative scheme

Figure 7 shows the pendulum position control on the physical platform once it has reached the stabilizable region between -15 and 15 degrees around the upward unstable position, starting from a swing-up behavior.

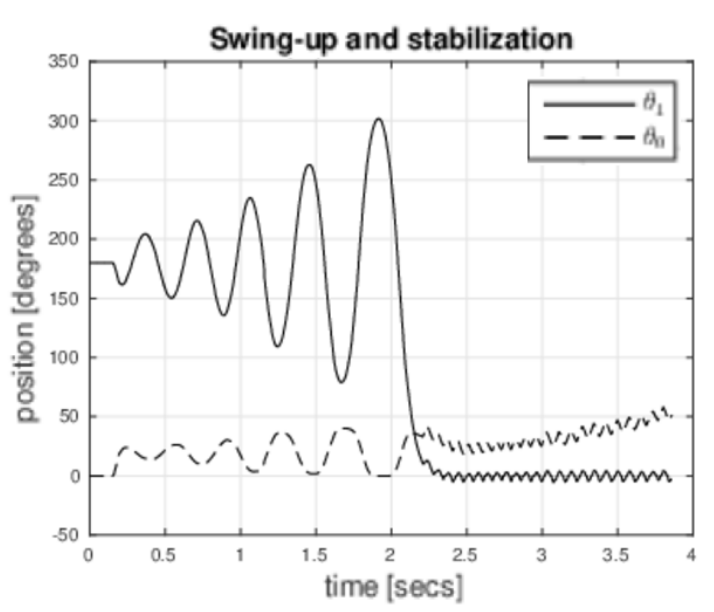

Figure 7. Pendulum position stabilization

Some switching strategies could include a hysteretic approach, that would define different thresholds for enabling the LQR controller to act within the linearizable region when $\left|\theta_{1}\right| \leq 15^{\circ}$, and then switch to the swing-up controller when the pendulum position moves to a downward position, as with $\left|\theta_{1}\right| \geq 20^{\circ}$. Although this approach could help prevent unwanted rapid switching, a single threshold for switching between controllers is considered, since it is not useful to allow the LQR controller to act within a non-linearizable region such as the pendulum position where $\sin \left(\theta_{1}\right)$ could not be approximated as $\theta_{1}$.

Although the pendulum position is appropriately controlled, Figure 7 shows that the arm position changes without any constraints in order to ensure that the pendulum keeps its upward unstable position. In order to be able to control both degrees of freedom of this underactuated plant in an experimental setting, the arm position (actuated link) has to be given more importance, so that $Q$ is modified accordingly:

$Q=\left[\begin{array}{ccccc}100 & 0 & 0 & 0 & 0 \\ 0 & 10 & 0 & 0 & 0 \\ 0 & 0 & 100 & 0 & 0 \\ 0 & 0 & 0 & 10 & 0 \\ 0 & 0 & 0 & 0 & 1\end{array}\right]$,

while the value of $R$ stays the same, $R=10$, leading to the solution of the ARE:

$P=\left[\begin{array}{ccccc}.0601 & .0153 & -.1718 & -.0216 & -.0002 \\ \bullet & .0141 & -.175 & -.0219 & -.0003 \\ \bullet & \bullet & 2.2155 & .2746 & .004 \\ \bullet & \bullet & \bullet & .0344 & .0005 \\ \bullet & \bullet & \bullet & \bullet & 0\end{array}\right] \cdot 10^{6}$ 
where $\mathrm{g}$ is used to denote symmetry. It is important to note that in order to keep (42) as small as possible when $Q$ has large values such as the ones in this last approach, the corresponding elements of the state have to be smaller. As it can be seen, this leads to the poles of the closed-loop system being moved further left in the complex plane so that the state decays faster to zero.

Thus, the matrix above generates the statefeedback controller described by

$$
L=\left[\begin{array}{lllll}
-3.1 & -6.07 & 80.02 & 10.08 & 0.19
\end{array}\right] \text {, }
$$

which yields closed-loop poles at

$$
\left\{\begin{array}{lllll}
-772 & -31 & -1.12 & -7.41+0.9 j & -7.41-0.9 j
\end{array}\right\}
$$

where $j$ denotes the complex component in the complex plane. The closed-loop stability with all its poles in the left-half complex plane can be noticed.
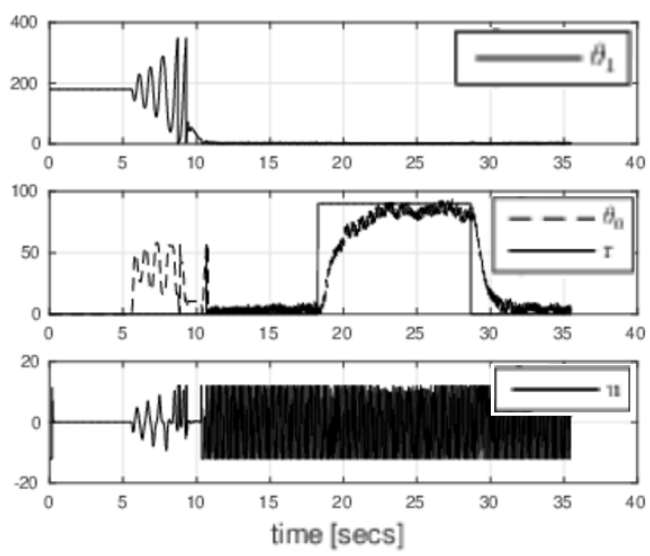

Figure 8. Pendulum and arm position control

Then, Figure 8 shows the signals of interest for the switched control strategy, which combines both the energy-based and LQR controllers for the swing-up and stabilization tasks respectively. The first plot shows the pendulum position, $\theta_{1}$, while the second plot shows tracking of reference $r$ for the actuated link, $\theta_{0}$, with an arbitrary change of $\frac{\pi}{2}$. Figure 8 also shows the control signal, $u$, which corresponds to the voltage applied to the DC motor. It can be noticed that oscillations in the actuation signal are caused by practical issues such as the zero position of the pendulum.

Therefore, if the vertical stable pendulum position is not exactly at 0 degrees and actuation is higher than desired, the pendulum will always be struggling against gravity.

\section{Conclusion}

A well-known underactuated system, namely the rotary inverted pendulum (also known as the Furuta pendulum) has been studied, and a linear model for the upward unstable position has been obtained. The switched control strategy presented involves an energy-based swing-up task and the stabilization of the pendulum in the upward position, based on a linear controller obtained by means of posing the stabilization problem as a linear quadratic regulator design. This has also proven to be of interest when controlling both degrees of freedom of the underactuated plant, since this paper is concerned with the simultaneous control of both the rotating arm and the inverted pendulum position of this physical prototype of the underactuated system. It is important to note that the linear quadratic regulator was designed in the continuous domain, which was controlled by a digital system (a computer with data acquisition card), which is possible when the sampling time is small enough in order to make a continuous approximation.

Further improvements to the controller structure and implementation are being sought in current work, in order to reduce the noise related to the control signal and to implement control techniques for the above-mentioned experimental prototype, specifically for feedback gain tuning. In addition, among other interesting approaches to be discussed in future works, there is the automatic adjustment of the reference signal, which is meant to tackle a change of environment where an initial zero measurement does not correspond to the upward unstable position of the pendulum.

\section{Acknowledgements}

This work was supported in part by: Fondecyt 1170123, in part by Basal Project FB0821, and in part by DGIP 23.15.26 


\section{REFERENCES}

1. Åström, K. J. \& Furuta, K. (2000). Swinging up a Pendulum by Energy Control, Automatica, 36(2), 287-295.

2. Block, D. J., Åström, K. J. \& Spong, M. W. (2007). The Reaction Wheel Pendulum. Morgan \& Claypool Publishers LLC.

3. Fantoni, I. \& Lozano, R. (2002). Non-Linear Control for Underactuated Mechanical Systems. Springer-Verlag.

4. Furuta, K., Yamakita, M. \& Kobayashi, S. (1992). Swing-up Control of Inverted Pendulum using Pseudo-State Feedback, Journal of Systems and Control Engineering, 206(4), 263-269.

5. Hassanzadeh, I. \& Mobayen, S. (2011). Controller Design for Rotary Inverted Pendulum System using Evolutionary Algorithms, Mathematical Problems in Engineering, Vol. 2011, Article ID 572424.

6. Jung, S. \& Cho, H. T. (2004). Decoupled Neural Network Reference Compensation Technique for a PD Controlled Two Degreesof-freedom Inverted Pendulum, International Journal of Control, Automation, and Systems, 2(1), 92-99.

7. Liberzon, D. (2003). Switching in Systems and Control. Springer Science \& Business Media.

8. Noh, J. S., Lee, G. H. \& Jung, S. (2010). Position Control of a Mobile Inverted Pendulum System using Radial Basis Function Network, International Journal of Control, Automation and Systems, 8(1), 157-162.

9. Olivares, M.\&Albertos, P. (2014).ASwitched Swing-up and Stabilization Control Strategy for the Rotating Flywheel Pendulum. In $11^{\text {th }}$ World Congress on Intelligent Control and Automation (pp. 3874-3880).

10. Olivares, M. \& Albertos, P. (2014). Linear Control of the Flywheel Inverted Pendulum, ISA Transactions, 53(5), 1396-1403.
11. Park, M., Kim, Y.-J. \& Lee, J.-J. (2011). Swing-up and LQR Stabilization of a Rotary Inverted Pendulum, Artificial Life and Robotics, 16(1), 94-97.

12. Ramírez-Neria, M., Sira-Ramírez, H., Garrido-Moctezuma, R. \& Luviano-Juarez, A. (2014). Linear Active Disturbance Rejection Control of Underactuated Systems: The Case of the Furuta Pendulum, ISA Transactions, 53(4), 920-928.

13. Seman, P., Juh, M. \& Salaj, M. (2013). Swinging up the Furuta Pendulum and its Stabilization via Model Predictive Control, Journal of Electrical Engineering, 64(3), 152-158.

14. Spong, M. W. (1998). Underactuated Mechanical Systems, Control Problems in Robotics and Automation, 135-150.

15. Yang, X. \& Zheng, X. (2018). Swing-up and Stabilization Control Design for an Underactuated Rotary Inverted Pendulum System: Theory and Experiments, IEEE Transactions on Industrial Electronics, 65(9), 7229-7238.

16. Yu, H., Liu, Y. \& Yang, T. (2008). ClosedLoop Tracking Control of a PendulumDriven Cart-Pole Underactuated System, Journal of Systems and Control Engineering, 222(2), 109-125.

17. Zehar, D., Benmahammed, K. \& Behih, K. (2018). Control for Underactuated Systems Using Sliding Mode Observer, International Journal of Control, Automation and Systems, 16(2), 739-748.

18. Zhang, M. \& Tarn, T.-J. (2003). A Hybrid Switching Control Strategy for Nonlinear and Underactuated Mechanical Systems, IEEE Transactions on Automatic Control, 48(10), 1777-1782. 